\title{
PATOGENICIDADE DE ISOLADOS DE TRÊS FUNGOS ENTOMOPATOGÊNICOS A SOLDADOS DE Atta sexdens sexdens (Linnaeus, 1758) (HYMENOPTERA: FORMICIDAE) ${ }^{1}$
}

\author{
Elisângela de Souza Loureiro² e Antonio Carlos Monteiro²
}

\begin{abstract}
RESUMO - As formigas cortadeiras atacam diversas culturas agrícolas, pastagens e os reflorestamentos, atuando sobre muitas espécies vegetais. O presente trabalho teve por objetivo avaliar a patogenicidade dos fungos Beauveria bassiana (isolados AM 9 e JAB 06), Metarhizium anisopliae (isolados E 9 e AL) e Paecilomyces farinosus (isolados CG 189 e CG 195) a soldados de Atta sexdens sexdens, em condições de laboratório. Após a coleta em formigueiro isento de produtos fitossanitários, exemplares de soldados foram cuidadosamente separados em grupos de oito indivíduos e, a seguir, banhados em suspensões contendo 1,0 x 10 $61,0 \times 10^{7}$, $1,0 \times 10^{8}$ e $1,0 \times 10^{9}$ conídios/mL de cada isolado. Em seguida, cada grupo de formigas foi transferido para unidades de câmara úmida e mantido, sem alimentação, a $27 \pm 1^{\circ} \mathrm{C}$. A mortalidade foi verificada diariamente. Os três fungos se mostraram eficientes patógenos, pois provocaram alta mortalidade, matando mais que $80 \%$ dos soldados nos quatro primeiros dias após a inoculação. Os melhores isolados foram JAB 06 e AL, na concentração de $1,0 \times 10^{9} \mathrm{con} . / \mathrm{mL}$. Os isolados JAB 06, AL, CG 195 apresentaram maior capacidade de esporular nos cadáveres das formigas e os tempos letais decresceram com o aumento da concentração de conídios usada.
\end{abstract}

Palavras-chave: Beauveria bassiana, Metarhiziun anisopliae, Paecilomyces farinosus, controle biológico, controle microbiano, formiga cortadeira e inseto social.

\section{PATHOGENICITY OF ISOLATES OF THREE ENTOMOPATHOGENIC FUNGI AGAINST SOLDIERS OF Atta sexdens sexdens (Linnaeus, 1758) (HYMENOPTERA: FORMICIDAE)}

\begin{abstract}
Leaf-cutting ants attack several crops, pastures and the planted forests, acting on numerous vegetable species. The present work aimed to evaluate the pathogenicity of the fungi Beauveria bassiana (isolates AM 9 and JAB O6), Metarhizium anisopliae (isolates $E 9$ and Al) and Paecilomyces farinosus (isolates CG 189 and CG 195) against soldiers of Atta sexdens sexdens, in laboratory conditions. These fungi were inoculated on soldiers after collection in nests without pesticides application. Specimens of soldiers were separated in groups of eight insects, which were bathed in suspensions containing $1.0 \times 10^{6}, 1.0 \times 10^{7}, 1.0$ $\times 10^{8}$ and $1.0 \times 10^{9}$ conidia/ml. Following the bath, each group of ants was taken to moist chamber units, and maintained for a period of starvation under at $27 \pm 1^{\circ} \mathrm{C}$. The mortality was updated on a daily basis. The three species of fungi were highly pathogenic to A. sexdens sexdens soldiers causing high mortality rates, above $80 \%$, killing the ants in the first four days after inoculation. The bests isolates were JAB 06 and AL,
\end{abstract}

\footnotetext{
${ }^{1}$ Recebido em 07.11.2003 e aceito para publicação em 20.04.2005.

${ }^{2}$ Laboratório de Microbiologia, Departamento de Produção Vegetal da Faculdade de Ciências Agrárias e Veterinárias da Unesp, Via de Acesso Prof. Paulo Donato Castellane, s/n - 14884-900 Jaboticabal-SP. E-mail: <lis_loureiro@ yahoo.com.br>; <montecar@fcav.unesp.br>.
} 
at the concentration $1.0 \times 10^{9} \mathrm{con} . / \mathrm{ml}$. The isolates JAB 06, AL and CG 195 showed higher capacity of spore production on ant cadavers and there was a decrease in lethal time with the increase in the spore concentration used.

Keywords: Beauveria bassiana, Metarhiziun anisopliae, Paecilomyces farinosus, biological control, leafcutting ant, microbial control, social insects.

\section{INTRODUÇÃO}

As formigas cortadeiras dos gêneros Atta e Acromyrmex são pragas agrícolas severas no Brasil. Distribuídas por todo o território nacional e com intensa atividade durante o ano, atacam várias culturas agrícolas, pastagens e, em particular, os reflorestamentos, atuando sobre muitas espécies vegetais (MARICONI, 1970; HERNANDEZ e JAFFÉ, 1995). Em reflorestamentos, atacam as plantas desde os viveiros até o campo definitivo (HERNANDEZ e JAFFÉ, 1995; ZANNETTI, 1998).

As empresas de reflorestamento têm empregado o controle químico de formigas cortadeiras de forma sistemática, através de iscas, termonebulização e fumigantes, sendo o aspecto econômico das operações de grande importância, em virtude dos altos custos envolvidos. Os produtos comerciais disponíveis até alguns anos atrás eram, de modo geral, formulados à base de inseticidas clorados, mas cuja fabricação foi suspensa pela legislação brasileira. O dodecacloro, princípio ativo mais usado até então, foi substituído pela sulfluramida, mas permaneceram os danos ecológicos. Além do alto custo ocasionado pelo uso de iscas à base de sulfluramida, os aspectos ambientais têm levado as empresas a investir na melhoria do rendimento operacional das técnicas de controle químico e na experimentação de novas tecnologias, objetivando minimizar os impactos ao meio ambiente.

Nesse contexto, uma alternativa para o controle das formigas saúvas é o controle biológico com o uso de fungos entomopatogênicos. O controle biológico é específico, permanente e econômico (DE BACH, 1968), pois, em geral, os gastos com a utilização de produtos biológicos são menores, em comparação com outros produtos. Entretanto, há poucas informações sobre o efeito desses fungos no controle de formigas cortadeiras ou formigas lava-pés (PEREIRA et al., 1993).

Alguns pesquisadores demonstraram que a ação desses agentes de controle de pragas varia em função da casta estudada e com a forma de sua aplicação (DIEHL-
FLEIG e SILVA, 1986; ALVES, 1998; DIEHL-FLEIG et al., 1988; SILVA e DIEHL-FLEIG, 1988; SPECHT et al., 1994). Indivíduos de determinada casta podem ser mais suscetíveis que os de outra, ou determinada espécie de fungo pode ser mais patogênica para soldados que para operárias (DIEHL-FLEIG et al., 1988; SPECHT et al., 1994; LOUREIRO, 1997).

O presente trabalho teve por objetivo avaliar a patogenicidade de isolados de Beauveria bassiana (Bals.) Vuill, Metarhizium anisopliae var. anisopliae (Metsch.) Sorokin e Paecilomyces farinosus (Holm ex SF Gray) Brown \& Smith para soldados de Atta sexdens sexdens (L., 1758) (Hymenoptera: Formicidae), visando conhecer as potencialidades destes fungos para o controle de formigas cortadeiras.

\section{MATERIAL E MÉTODOS}

Os fungos utilizados nos testes de patogenicidade foram os isolados AM 9 e JAB 06 de B. bassiana, E 9 e AL de M. anisopliae e CG 189 e CG 195 de P. farinosus.

Os isolados foram mantidos em tubos de ensaio contendo $5 \mathrm{~mL}$ de BDA (batata-dextrose-ágar) sólido inclinado e armazenados em refrigerador a $4{ }^{\circ} \mathrm{C}$. Para utilização nos ensaios foram multiplicados em placas de Petri contendo BDA, pelo método de espalhamento. Após a incubação a $27 \pm 1{ }^{\circ} \mathrm{C}$, umidade relativa do ar acima de $80 \%$ e fotofase de 12 horas por 10 dias, os conídios formados na superfície da colônia foram coletados com o auxílio de alça de níquel-cromo previamente flambada e transferidos para tubos de ensaio contendo $10 \mathrm{~mL}$ de solução aquosa de Tween 80 a $0,1 \%$ (v/v). Após vigorosa agitação para desagregação das cadeias, procedeu-se à contagem do número de conídios, ao microscópio óptico, através da câmara de Neubauer.

Utilizaram-se soldados de Atta sexdens sexdens coletados de sauveiro isento da aplicação de produtos fitossanitários, em área de reflorestamento de Eucaliptus spp. pertencente à Fazenda de Ensino e Pesquisa da Faculdade de Ciências Agrárias e Veterinárias da Unesp, Campus de Jaboticabal, SP. 
Os exemplares de soldados foram coletados com o auxílio de pinça entomológica, em dias de intensa atividade no sauveiro, colocados em frascos de vidro com tampa telada e trazidos para o laboratório. Grupos de oito formigas foram envoltas em pedaço de filó e banhadas, por 10 segundos, em suspensões de conídios de cada um dos isolados, com as concentrações de $1,0 \times 10^{6}, 1,0 \times 10^{7}, 1,0 \times 10^{8}$ e $1,0 \times 10^{9} \mathrm{con} . / \mathrm{mL}$ e um tratamento contendo apenas a solução de Tween 80 (testemunha). Em seguida, foram transferidas para câmaras úmidas (MONTEIRO, 1988) incubadas a $27 \pm 1^{\circ} \mathrm{C}$, umidade relativa do ar acima de $80 \%$ (SOLOMON, 1945), no escuro, por até 15 dias. Os insetos foram mantidos sem alimentação, e para verificação da mortalidade as câmaras foram inspecionadas diariamente. Os insetos mortos foram desinfectados superficialmente com álcool $70^{\circ} \mathrm{GL}$ e água destilada esterilizada e transferidos para novas câmaras, mantidas em iguais condições, visando à verificação da extrusão do patógeno para confirmação da mortalidade. A esporulação dos fungos sobre os cadáveres das formigas foi avaliada 15 dias após a inoculação.

Utilizou-se um experimento fatorial $3 \times 2 \times 6$ (fungos $\mathrm{x}$ isolados $\mathrm{x}$ concentrações) no delineamento inteiramente casualizado. Para cada tratamento, realizaram-se três repetições (câmaras úmidas) contendo oito formigas cada repetição. Além do grupo-controle em que as formigas foram banhadas apenas na solução de Tween 80 , efetuouse outro tratamento-testemunha, sem banho.

Os dados referentes à mortalidade e à esporulação foram submetidos à análise de variância e as médias de tratamentos, comparadas pelo teste de Tukey a 5\% de probabilidade. Na análise de regressão, a probabilidade de erro foi de $1 \%$. Para obtenção dos valores de $\mathrm{TL}_{50}$ (em dias) foi realizada a análise de Probit nos diversos tratamentos.

\section{RESULTADOS E DISCUSSÃO}

\subsection{Patogenicidade dos isolados dos fungos a soldados de Atta sexdens sexdens}

A ação patogênica dos fungos foi evidenciada a partir do $2^{\circ}$ dia após a inoculação, provocando mortalidade superior à observada nos tratamentostestemunha, e estendeu-se até o $4^{\circ}$ dia após a inoculação, ocorrendo diferença estatística entre eles (Quadro 1). No $5^{\circ}$ e $6^{\circ}$ dias, a mortalidade ocorrida nos tratamentos com os fungos praticamente se igualou àquela verificada nas testemunhas. A partir do $7^{\circ}$ dia, a patogenicidade dos fungos deixou de ser a razão determinante da morte das formigas, em razão da maior mortalidade observada nos tratamentos-testemunhas. Contudo, isso ocorreu porque os três fungos foram virulentos, matando, nos quatro primeiros dias, mais de $80 \%$ das formigas utilizadas nos ensaios (Quadro 1). Verificou-se diferença estatística nos tratamentos, em comparação com os valores da testemunha (Quadro 1). Diehl-Fleig et al. (1993) obtiveram $87,2 \%$ de mortalidade de Acromyrmex crassipinus e A. heyeri decorridos 35 dias da aplicação, em colônias no campo, de $1,0 \times 10^{9}$ con./0,1 g de arroz esporulado de B. bassiana, isolado $\mathrm{B}_{\mathrm{sa}}$.

B. bassiana foi o fungo mais patogênico dentre os avaliados, sendo observado um $\mathrm{TL}_{50}$ de 2,60 e 2,72 dias para os isolados JAB 06 e AM 9, respectivamente (Quadro 2). Alves e Soza Gómez (1983) também observaram maior agressividade de $B$. bassiana que M. anisopliae em Atta sexdens rubropilosa, o que foi confirmado no presente trabalho.

Analisando a patogenicidade dos isolados, em função do tempo após a inoculação, observou-se que todos os isolados foram altamente virulentos. A mortalidade dos soldados tratados com os isolados AM 9 de $B$. bassiana $\left(\mathrm{r}^{2}=0,94 ; \mathrm{F}=46,66\right)$, E $9\left(\mathrm{r}^{2}=0,98\right.$; $\mathrm{F}=164,53)$, AL de M. anisopliae $\left(\mathrm{r}^{2}=0,95 ; \mathrm{F}=53,11\right)$ e CG $189\left(\mathrm{r}^{2}=0,98 ; \mathrm{F}=137,13\right)$ de $P$. farinosus, em função do tempo após a inoculação, ocorreu pelo modelo quadrático (Figuras 1A, 2AB e 3A). A mortalidade máxima de formigas com o tratamento ocorreu entre o $4^{\circ}$ e o $6^{\circ}$ dia após a sua imersão nas suspensões contendo os esporos, e até o $8^{\circ}$ dia não houve alteração significativa nessas mortes (Figuras 1A, 2A, 2B e 3A). Todavia, em soldados tratados com os isolados JAB 06 de B. bassiana e CG 195 de $P$. farinosus, a mortalidade aconteceu seguindo o modelo cúbico $\left(\mathrm{r}^{2}=0,99 ; \mathrm{F}=\right.$ 320,32 ), com morte máxima de formigas (100\%) ocorrendo até $04^{\circ}$ dia depois da inoculação, seguida de estabilização da curva, porque, consequientemente, não houve mais mortes. Usando o isolado 447 de B. bassiana em operárias de $S$. invicta, Pereira et al. (1993) obtiveram $100 \%$ de infecção e mortalidade. Stimac et al. (1993) verificaram que o tratamento de colônias de $S$. invicta, em laboratório, com 1 e 2 g de esporos de B. bassiana matou menos de $90 \%$ das formigas.

R. Árvore, Viçosa-MG, v.29, n.4, p.553-561, 2005 


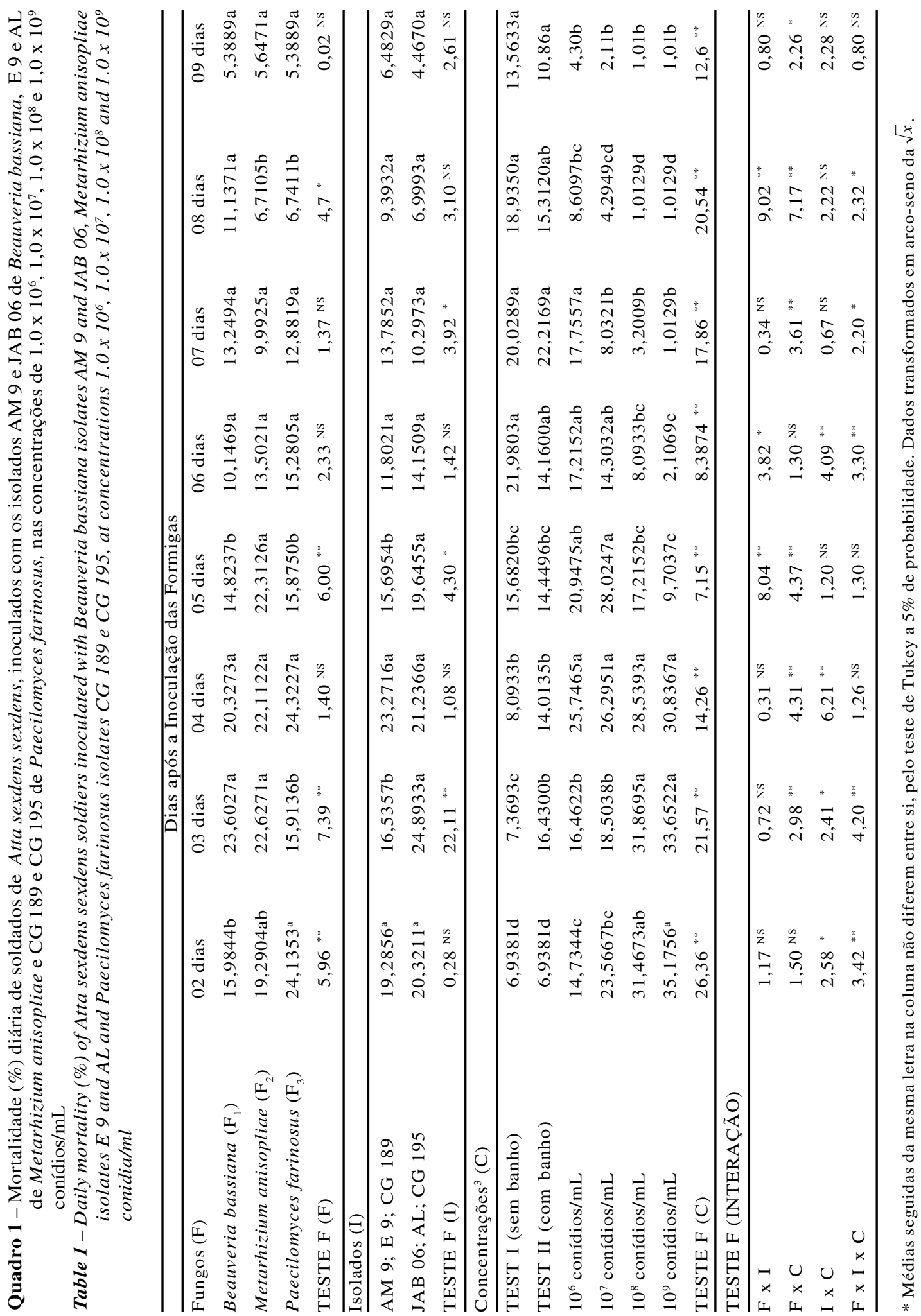


Quadro 2 - Tempos letais médios $\left(\mathrm{TL}_{50}\right)$, equações de regressão linear e valores de $\mathrm{x}^{2}$ obtidos pela análise de Probit para concentrações de conídios dos isolados de Beauveria bassiana (AM 9 e JAB 06), Metarhizium anisopliae (E 9 e AL) e Paecilomyces farinosus (CG 189 e CG 195) para soldados de Atta sexdens sexdens

Table 2 - Mean lethal time $\left(L T_{50}\right)$, regression linear equation and $x^{2}$ value obtained by Probit's analysis for conidial concentration of isolates of Beauveria bassiana (AM 9 and JAB O6), Metarhizium anisopliae ( 9 and A L) and Paecilomyces farinosus (CG 189 and CG 195) for Atta sexdens sexdens soldiers

\begin{tabular}{|c|c|c|c|c|}
\hline Fungo/Isolado/Concentração & $\mathrm{TL}_{50}$ (dias) & IC & Modelo Linear & $\mathrm{x}^{2}$ \\
\hline \multicolumn{5}{|l|}{$\overline{\text { B. bassiana (AM 9) }}$} \\
\hline $10^{6}$ conídios $/ \mathrm{mL}$ & 5,22 & $(4,69-5,81)$ & $y^{\prime}=0,165+6,735^{*} \log x$ & 4,34 \\
\hline $10^{7}$ conídios $/ \mathrm{mL}$ & 2,79 & $(2,41-3,25)$ & $y^{\prime}=2,268+6,122 * \log x$ & 1,84 \\
\hline $10^{8}$ conídios $/ \mathrm{mL}$ & 2,72 & $(2,22-3,35)$ & $y^{\prime}=2,745+5,180 * \log x$ & 2,55 \\
\hline $10^{9}$ conídios $/ \mathrm{mL}$ & 2,75 & $(2,13-3,55)$ & $y^{\prime}=1,206+8,634 * \log x$ & 3,06 \\
\hline \multicolumn{5}{|l|}{$\overline{\text { B. bassiana (JAB 06) }}$} \\
\hline $10^{6}$ conídios $/ \mathrm{mL}$ & 5,41 & $(4,99-5,86)$ & $y^{\prime}=-6,508+15,70 * \log x$ & 5,12 \\
\hline $10^{7}$ conídios $/ \mathrm{mL}$ & 4,06 & $(3,81-4,34)$ & $y^{\prime}=-2,097+11,65 * \log x$ & 1,09 \\
\hline $10^{8}$ conídios $/ \mathrm{mL}$ & 2,97 & $(2,31-3,83)$ & $y^{\prime}=-0,081+10,74 * \log x$ & 4,04 \\
\hline $10^{9}$ conídios $/ \mathrm{mL}$ & 2,60 & $(2,00-3,37)$ & $y^{\prime}=2,434+6,189 * \log x$ & 1,80 \\
\hline \multicolumn{5}{|l|}{ M. anisopliae (E9) } \\
\hline $10^{6}$ conídios $/ \mathrm{mL}$ & 6,02 & $(5,61-6,46)$ & $y^{\prime}=-5,282+13,19 * \log x$ & 3,70 \\
\hline $10^{7}$ conídios $/ \mathrm{mL}$ & 4,98 & $(4,65-5,33)$ & $\mathrm{y}^{\prime}=-3,582+12,31 * \log \mathrm{x}$ & 2,67 \\
\hline $10^{8}$ conídios $/ \mathrm{mL}$ & 2,77 & $(2,30-3,33)$ & $y^{\prime}=2,491+5,673 * \log x$ & 2,40 \\
\hline $10^{9}$ conídios $/ \mathrm{mL}$ & 2,77 & $(2,34-3,29)$ & $\mathrm{y}^{\prime}=1,702+7,440 * \log \mathrm{x}$ & 1,15 \\
\hline \multicolumn{5}{|l|}{$\overline{\text { M. anisopliae (AL) }}$} \\
\hline $10^{6}$ conídios $/ \mathrm{mL}$ & 3,80 & $(3,34-4,33)$ & $y^{\prime}=0,355+8,003 * \log x$ & 4,73 \\
\hline $10^{7}$ conídios $/ \mathrm{mL}$ & 3,71 & $(3,53-3,90)$ & $y^{\prime}=-1,031+10,59 * \log x$ & 0,54 \\
\hline $10^{8}$ conídios $/ \mathrm{mL}$ & 3,05 & $(2,72-3,41)$ & $\mathrm{y}^{\prime}=-0,767+11,92 * \log \mathrm{x}$ & 0,90 \\
\hline $10^{9}$ conídios $/ \mathrm{mL}$ & 2,69 & $(2,64-2,74)$ & $y^{\prime}=0,861+9,626^{*} \log x$ & 0,02 \\
\hline \multicolumn{5}{|l|}{$\overline{P . \text { farinosus }(\mathrm{CG} 189)}$} \\
\hline $10^{6}$ conídios $/ \mathrm{mL}^{1}$ & - & _ & - & _- \\
\hline $10^{7}$ conídios $/ \mathrm{mL}^{1}$ & - & - & - & - \\
\hline $10^{8}$ conídios $/ \mathrm{mL}^{1}$ & - & - & - & - \\
\hline $10^{9}$ conídios $/ \mathrm{mL}$ & 2,98 & $(2,26-3,93)$ & $y^{\prime}=1,432+7,524 * \log x$ & 3,27 \\
\hline \multicolumn{5}{|l|}{ P. farinosus (CG 195) } \\
\hline $10^{6}$ conídios $/ \mathrm{mL}^{1}$ & - & - & - & - \\
\hline $10^{7}$ conídios $/ \mathrm{mL}^{1}$ & - & - & - & - \\
\hline $10^{8} \operatorname{conídios} / \mathrm{mL}^{1}$ & - & - & - & - \\
\hline $10^{9}$ conídios $/ \mathrm{mL}$ & 3,14 & $(2,30-4,27)$ & $y^{\prime}=1,036+7,989 * \log x$ & 4,63 \\
\hline
\end{tabular}

${ }^{1}$ A taxa de mortalidade na testemunha foi superior a $50 \%$, não permitindo o cálculo da mortalidade na análise de Probit no período estudado.
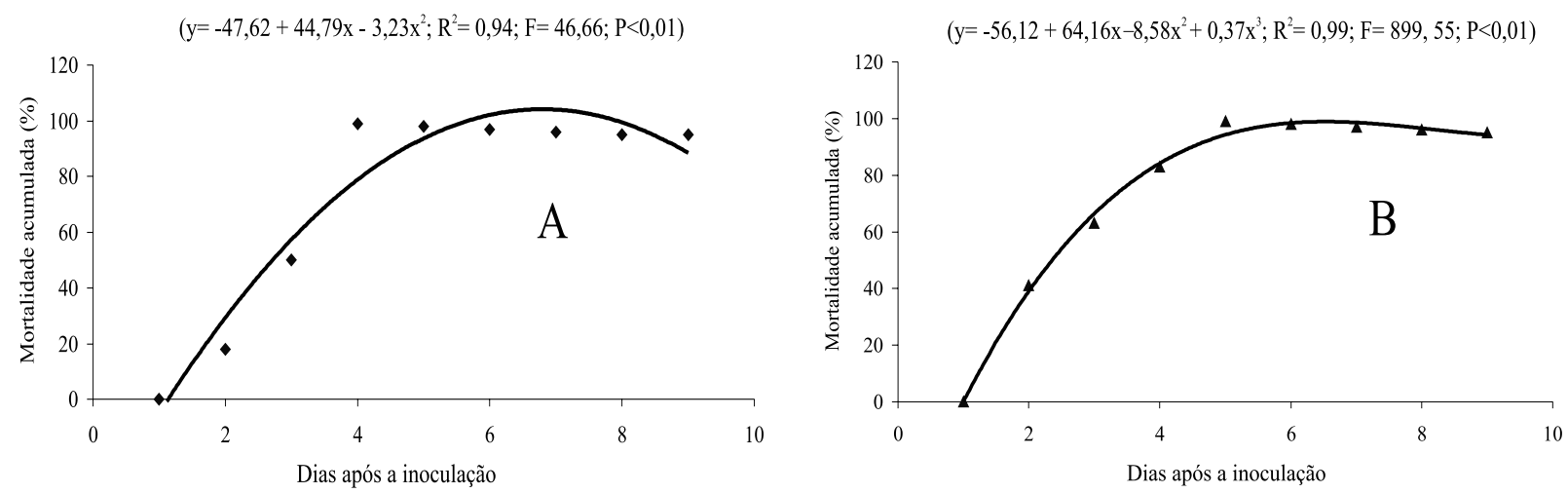

Figura 1 - Mortalidade acumulada (\%) de soldados de Atta sexdens sexdens, em função dos isolados AM 9 (A) e JAB 06 (B) de Beauveria bassiana e do tempo após a inoculação das formigas.

Figure 1 - Cumulative mortality (\%) of Atta sexdens sexdens soldiers, in function of isolates AM 9 (A) and JAB O6 (B) of Beauveria bassiana and the time after inoculation. 

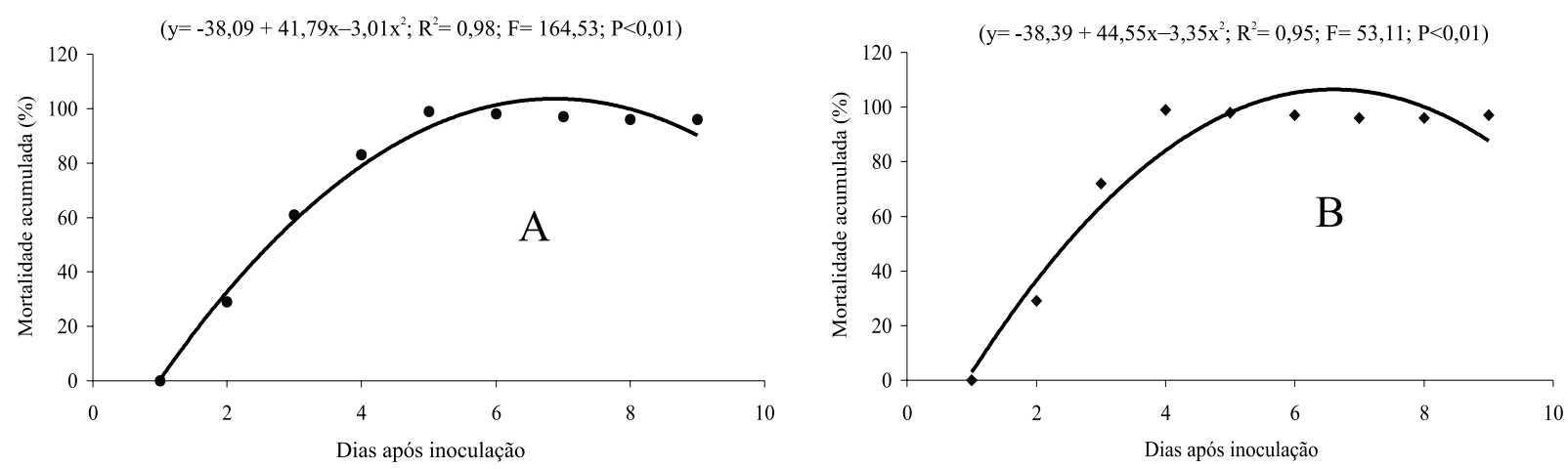

Figura 2 - Mortalidade acumulada (\%) de soldados de Atta sexdens sexdens, em função dos isolados E 9 (A) e AL (B) de Metarhizium anisopliae e do tempo após a inoculação das formigas.

Figure 2 - Cumulative mortality (\%) of Atta sexdens sexdens soldiers, in function of the isolates E $9(A)$ and $A L(B)$ of Metarhizium anisopliae and the time after inoculation.
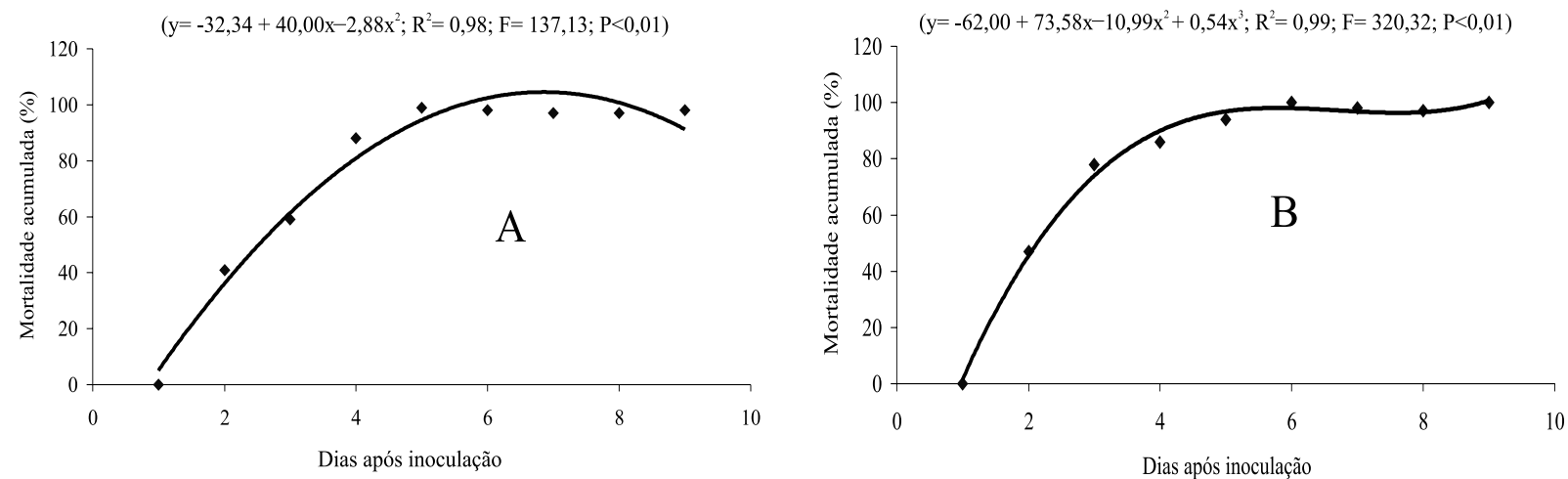

Figura 3 - Mortalidade acumulada (\%) de soldados de Atta sexdens sexdens, em função dos isolados CG 189 (A) e CG 195 (B) de Paecilomyces farinosus e do tempo após a inoculação das formigas.

Figure 3 - Cumulative mortality (\%) of Atta sexdens sexdens soldiers, in function of the isolates CG 189 (A) and CG 195 (B) of Paecilomyces farinosus and the time after inoculation.

Através das análises de Probit, determinaram-se os tempos letais médios de mortalidade, ocorridos em função das diferentes concentrações de conídios usadas em cada isolado (Quadro 2). Observou-se que, quando os soldados foram infectados pelo isolado AM 9 de $B$. bassiana, não ocorreu diferença significativa entre as concentrações de $1,0 \times 10^{9}, 1,0 \times 10^{8}$ e $1,0 \times 10^{7}$ con./mL, com base na sobreposição dos intervalos de confiança obtidos, com pequena diferença no tempo letal das concentrações $(2,75,2,72$ e 2,79 dias, respectivamente). Os isolados AM 9 e JAB 06 provocaram 50\% de mortalidade da população de formigas em tempo inferior a três dias, exceto a menor concentração em ambos os isolados e a concentração de $1,0 \times 10^{7} \mathrm{con} . / \mathrm{mL}$ de
JAB 06 (4,06 dias) (Quadro 2). Com JAB 06 de B. bassiana e E 9 de $M$. anisopliae ocorreu diferença entre as concentrações, exceto a concentração de 1,0 x $10^{9} \mathrm{con} . / \mathrm{mL}$.

Os tempos letais medianos desse experimento foram menores que os obtidos por Pereira et al. (1993), para o isolado 447 de B. bassiana, em operárias de $S$. invicta, sendo de cinco a seis dias para a concentração de $10^{8} \mathrm{con} . / \mathrm{mL}$ e de seis a oito dias para $10^{7} \mathrm{con} . / \mathrm{mL}$. DielhFleig e Silva (1986) utilizaram, em laboratório, suspensões de conídios do isolado $\mathrm{L}_{87}$ de $B$. bassiana, na concentração de $10^{6}$ con./mL, obtendo um $\mathrm{TL}_{50}$ de 3,44 dias. Esse valor é menor que os obtidos nos isolados AM 9 e JAB 06, na mesma concentração. Contudo, no isolado AL de $M$. anisopliae observou-se um $\mathrm{TL}_{50}$ 
de 2,69 dias, na concentração de $1,0 \times 10^{9} \mathrm{con} . / \mathrm{mL}$, e de 3,80 dias na de $1,0 \times 10^{6} \mathrm{con} . / \mathrm{mL}$ (Quadro 2). Esses dados são semelhantes aos de Dielh-Fleig e Silva (1986), que, utilizando o isolado AL e a concentração de 1,0 x $10^{9}$ con./mL, obtiveram um $\mathrm{TL}_{50}$ de 3,00 dias. Alves e Sosa-Gomez (1983) testaram a patogenicidade de uma linhagem de B. bassiana e de $M$. anisopliae, isoladas de rainha de $A$. sexdens rubropilosa, sobre soldados e cortadeiras dessa mesma espécie. Constataram que ambos os fungos foram patogênicos à formiga, sendo os soldados mais sensíveis a B. bassiana e as cortadeiras, a M. anisopliae.

Nos isolados de P. farinosus, o mesmo desempenho não foi observado, já que a mortalidade na testemunha, passados oito a nove dias de incubação, alcançou valores superiores a $50 \%$, dificultando o estudo dessa característica no período de avaliação. Entretanto, os isolados CG 189 e CG 195 foram os menos virulentos, sendo possível calcular apenas o $\mathrm{TL}_{50}$ da concentração de $1,0 \times 10^{9} \mathrm{con} . / \mathrm{mL}$, com 2,98 e 3,14 dias, respectivamente (Quadro 2).

Embora não tenham ocorrido diferenças entre os isolados testados, exceto no $3^{\circ}$ e $5^{\circ}$ dias (Quadro1), pôde-se observar, através dos $\mathrm{TL}_{50}$, que os isolados JAB 06, ALe CG 189 provocaram mortalidade de soldados em menor espaço de tempo $(2,60 ; 2,69$; e 2,98 dias, respectivamente) (Quadro 2). Segundo Diehl-Fleig et al. (1988), a virulência entre isolados, inclusive de mesma espécie de fungos, é variável.

Em todas as concentrações de conídios utilizadas, o qui-quadrado $\left(x^{2}\right)$ não foi significativo, indicando que essa característica pode ser estudada por esse método. De maneira geral, os tempos letais foram decrescentes à medida que aumentou a concentração de conídios (Quadro 2).

\subsection{Esporulação dos isolados sobre os cadáveres de soldados de Atta sexdens sexdens}

No grupo de formigas em que foi aplicado apenas o veículo das suspensões de conídios (testemunha), não ocorreram extrusão e esporulação dos fungos sobre os cadáveres das formigas cortadeiras, indicando que a mortalidade ocorrida nesse tratamento não foi resultado da infecção pelo fungo (STIMAC et al., 1987; ALVES et al., 1988; STIMAC et al., 1993; LOUREIRO, 1997). Entretanto, nos tratamentos em que foram aplicados os isolados observou-se alta porcentagem de extrusão dos fungos sobre o cadáver dos soldados.
Os três fungos apresentaram capacidade semelhante de crescer em cadáveres de formigas (Quadro 3). Todavia, houve diferenças entre isolados quanto a essa mesma característica, tendo os isolados JAB 06 (B. bassiana), AL (M. anisopliae) e CG 195 (P. farinosus) mostrado maior capacidade de esporular em cadáveres de $A$. sexdens sexdens que os isolados AM 9 (B. bassiana), E 9 (M. anisopliae) e CG 189 ( $P$. farinosus). O isolado 447 de $B$. bassiana esporulou sobre $88 \%$ dos cadáveres de operárias de $S$. invicta (PEREIRA et al., 1993), dado esse superior ao encontrado no presente trabalho $(41,67 \%)$.

Quadro 3-Esporulação (\%) dos isolados de Beauveria bassiana, Metarhizium anisopliae e Paecilomyces farinosus observada em cadáveres de soldados de Atta sexdens sexdens

Table 3 - Sporulation (\%) of isolates of Beauveria bassiana, Metarhizium anisopliae and Paecilomyces farinosus, observed in cadavers of Atta sexdens sexdens soldiers

\begin{tabular}{|c|c|}
\hline TRATAMENTOS & $\begin{array}{c}\text { Esporulação Observada } \\
15 \text { dias após a } \\
\text { Inoculação das Formigas }{ }^{1}\end{array}$ \\
\hline \multicolumn{2}{|l|}{ Fungos ${ }^{1}(\mathrm{~F})$} \\
\hline Beauveria bassiana $\left(\mathrm{F}_{1}\right)$ & $41,6667 a$ \\
\hline Metarhizium anisopliae $\left(\mathrm{F}_{2}\right)$ & $45,1389 \mathrm{a}$ \\
\hline Paecilomyces farinosus $\left(\mathrm{F}_{3}\right)$ & $50,0000 \mathrm{a}$ \\
\hline TESTE F (F) & $1,35 \mathrm{NS}$ \\
\hline DMS $(5 \%)$ & 18,1828 \\
\hline \multicolumn{2}{|l|}{$\overline{\text { Isolados }^{2}(\mathrm{I})}$} \\
\hline AM 9; E 9; CG 189 & $40,0463 b$ \\
\hline JAB 06; AL; CG 195 & $51,1574 a$ \\
\hline TESTE F (I) & $7,15^{* *}$ \\
\hline DMS $(5 \%)$ & 8,2828 \\
\hline \multicolumn{2}{|l|}{ Concentrações (C) } \\
\hline TEST I (sem banho) & $0,0000 \mathrm{c}$ \\
\hline TEST II (com banho) & $0,0000 \mathrm{c}$ \\
\hline $10^{6}$ conídios $/ \mathrm{mL}$ & $52,7778 b$ \\
\hline $10^{7}$ conídios $/ \mathrm{mL}$ & $71,5278 \mathrm{ab}$ \\
\hline $10^{8}$ conídios $/ \mathrm{mL}$ & $76,3889 a$ \\
\hline $10^{9}$ conídios $/ \mathrm{mL}$ & $72,9167 \mathrm{ab}$ \\
\hline TESTE F (C) & $50,82^{* *}$ \\
\hline DMS $(5 \%)$ & 21,0615 \\
\hline \multicolumn{2}{|l|}{ TESTE F (INTERAÇÃO) } \\
\hline $\mathrm{F} \times \mathrm{I}$ & $0,26 \mathrm{Ns}$ \\
\hline $\mathrm{F} \times \mathrm{C}$ & $2,02 *$ \\
\hline $\mathrm{I} \times \mathrm{C}$ & $1,48^{\mathrm{NS}}$ \\
\hline $\mathrm{F} \times \mathrm{I} \times \mathrm{C}$ & $0,44^{\mathrm{NS}}$ \\
\hline
\end{tabular}

Médias seguidas da mesma letra em cada coluna não diferem estatisticamente, pelo teste de Tukey a $5 \%$ de probabilidade (dados transformados em arco-seno $\sqrt{P / 100}$, em que $\mathrm{P}$ é a porcentagem de mortalidade de soldados).

${ }^{2} \mathrm{AM} 9$ e JAB 06 - Beauveria bassiana, E 9 e AL - Metarhizium anisopliae, CG 189 e CG 195 - Paecilomyces farinosus.

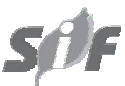

R. Árvore, Viçosa-MG, v.29, n.4, p.553-561, 2005 
A esporulação em cadáveres de seus hospedeiros é uma prova da capacidade do fungo em penetrar e colonizar, causando-lhes, então, a morte (DIEHL-FLEIG et al., 1988).

Os fungos avaliados foram patogênicos para soldados de Atta sexdens sexdens. Os tempos letais decresceram com o aumento da concentração de conídios usada. O isolado JAB 06 de B. bassiana foi o mais eficaz, provocando maior mortalidade na concentração de $1,0 \times 10^{9} \mathrm{con} . / \mathrm{mL}$, menor tempo letal e eficiente esporulação sobre cadáveres de soldados. O isolado AL de M. anisopliae também produziu alta mortalidade e eficiente esporulação, quando usado na mesma concentração de conídios, e CG 195 ( $P$. farinosus) apresentou boa capacidade de esporular sobre os cadáveres das formigas.

Os fungos avaliados mostraram a potencialidade como agentes de controle de soldados de A. sexdens sexdens. A grande variabilidade genética presente em fungos entomopatogênicos (ALVES, 1998) pode ser um dos principais fatores responsáveis pelas diferenças de virulência entre isolados e espécies, como referido por Diehl-Fleig et al. (1988). Novos estudos em laboratório e campo, envolvendo diferentes espécies e isolados, concentrações de esporos, castas de formigas e formas de aplicação, necessitam ser conduzidos para melhor aferir a eficiência dos fungos como agentes controladores desses insetos em condições de campo.

\section{REFERÊNCIAS BIBLIOGRÁFICAS}

ALVES, S.B.; SOSA GÓMEZ, D.R. Virulência do Metarhizium anisopliae (Metsch.) Sorok e Beauveria bassiana (Bals.) Vuill. para duas castas de Atta sexdens rubropilosa (Forel, 1968). Poliagro, v.5, n.1, p.1-9, 1983.

ALVES, S. B. Fungos entomopatogênicos In: ALVES, S. B. (Ed.). Controle microbiano de insetos. Piracicaba: Escola Superior Agricultura Luiz de Queiroz, 1998. p. 289-381.

ALVES, S.B.; STIMAC, J.C.; CAMARGO, M.T.V. Suscetibilidade de Solenopsis invicta Buren and $S$. saevissima FR. Smith. a isolados de $B$. bassiana (Bals.) Vuill. Anais da Sociedade Entomológica do Brasil, v.17, n.2, p.379-87, 1988.

R. Árvore, Viçosa-MG, v.29, n.4, p.553-561, 2005
DE BACH, P. Control biologico de las plagas de insetos $y$ malas hierbas. México, D.F.: Compañia Editorial Continental, 1968. 949p.

DIEHL-FLEIG, E.; SILVA, M.E. Patogenicidade de Beauveria bassiana e Metarhizium anisopliae à formiga saúva Atta sexdens piriventris. Boletim do Grupo de Pesquisadores de Controle Biológico, n.6, p.15, 1986.

DIEHL-FLEIG, E.; SILVA, M.E.; PACHECO, M.R.M. Testes de patogenicidade dos fungos entomopatogênicos Beauveria bassiana e Metarhizium anisopliae em Atta sexdens piriventris (Santschi, 1919) em diferentes temperaturas. Ciência e Cultura, v.40, n.11, p.1103-1105, 1988.

DIEHL-FLEIG, E. et al. Efficiency of Beauveria bassiana for Acromyrmex spp. control (Hymenoptera: Formicidae). Anais da Sociedade Entomológica do Brasil, v.22, n.2, p.281-285, 1993.

HERNANDEZ, J.V.; JAFFÉ, K. Dano econômico causado por populações de formigas Atta laevigata (F. Smith) em plantações de Pinus caribaea Mor. Elementos para o manejo da praga. Anais da Sociedade Entomológica do Brasil, v.24, n.2, p.287-298, 1995.

LOUREIRO, E.S. Patogenicidade de isolados dos fungos Beauveria bassiana, Metarhizium anisopliae e Paecilomyces farinosus sobre Atta sexdens sexdens (Linnaeus, 1758) em condições de laboratório. Jaboticabal: Universidade Estadual Paulista, 1997. 70p. (Trabalho de Graduação em Agronomia).

MARICONI, F.A.M. As saúvas. São Paulo: Agronômica Ceres, 1970. 167p.

MOnTEiro, A.C. Aspectos fisioecológicos de isolados de fungos entomopatogênicos obtidos na região amazônica (Manaus). 1988. 233f. Tese (Doutorado em Ecologia e Recursos Naturais). Universidade Federal de São Carlos, São Carlos, 1988.

PEREIRA, R.M.; ALVES, S.B.; STIMAC, J.L.

Growth of Beauveria bassiana in fire ant nest soil with amendments. Journal of Invertebrate Pathology, v.62, n.1, p.9-14, 1993.

R. Árvore, Viçosa-MG, v.29, n.4, p.553-561, 2005 
SILVA, M.E.; DIEHL-FLEIG, E. Avaliação de diferentes linhagens de fungos

entomopatogênicos para controle da formiga Atta sexdens piriventris (Santschi, 1919)

(Hymenoptera: Formicidae). Anais da

Sociedade Entomológica do Brasil, v.17, n.2, p.263-269, 1988.

SOLOMON, M.E. The use of cobalt salts as indicators of humidity and moisture. Annual Applied Biology, v.32, p.75-85, 1945.

SPECHT, A.; DIEHL-FLEIG, E.; SILVA, M.E.

Atratividade de iscas de B. bassiana (Bals) Vuill. a formiga do gênero Acromyrmex (Hymenoptera:

Formicidae). Anais da Sociedade

Entomológica do Brasil, v.23, n.1, p.99-104, 1994.
STIMAC, J.C.; PEREIRA, R.M.; ALVES, S.B. Suscetibilidade de Solenopsis spp a diferentes espécies de fungos entomopatogênicos. Anais da Sociedade Entomológica do Brasil, v.16, p.377-380, 1987.

STIMAC, J.L.; ALVES, S.B.; PEREIRA, R.M Mortality in laboratory colonies of Solenopsis invicta (Hymenoptera: Formicidae) treated with Beauveria bassiana (Deuteromycetes). Journal of Economic Entomology, v.86, n.4, p.10831087, 1993.

ZANNETTI, R. Estimativa do nível de dano econômico causado por formigas cortadeiras em eucaliptais. 1998. 85f. Tese (Doutorado em Ciência Florestal) - Universidade Federal de Viçosa, Viçosa, 1998. 